\title{
Belphégor
}

\section{Introduction. Production de l'intérêt grivélien : Fantastique maître Charles.}

\section{Matthieu Letourneux and Jacques Migozzi}

\section{(2) OpenEdition}

1 Journals

\section{Electronic version}

URL: https://journals.openedition.org/belphegor/1228

DOI: 10.4000/belphegor.1228

ISSN: 1499-7185

Publisher

LPCM

\section{Electronic reference}

Matthieu Letourneux and Jacques Migozzi, "Introduction. Production de l'intérêt grivélien : Fantastique maître Charles.", Belphégor [Online], 16-1 | 2018, Online since 27 March 2019, connection on 19 October 2021. URL: http://journals.openedition.org/belphegor/1228 ; DOI: https://doi.org/10.4000/ belphegor.1228

This text was automatically generated on 19 October 2021.

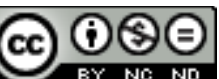

Belphégor est mis à disposition selon les termes de la Licence Creative Commons Attribution - Pas d'Utilisation Commerciale - Pas de Modification 4.0 International. 


\title{
Introduction. Production de l'intérêt grivélien : Fantastique maître Charles.
}

\author{
Matthieu Letourneux and Jacques Migozzi
}

1 Charles Grivel, notre ami, nous a quittés voici trois ans. Sa parole singulière, joueuse, provocatrice, stimulante, nous manque. Par ce numéro spécial de Belphégor, où sa voix alternera avec celle de partenaires qui l'ont connu et qu'il a inspirés, nous souhaitons rendre hommage à son audace de franc-tireur, d'accélérateur de questionnements et de défricheur de chemins de traverse.

2 Ce dossier n'aura nullement la prétention de rendre compte de tout l'empan des investigations éclectiques de Charles : son travail au très long cours sur les écrivains naturalistes ou sur la littérature Fin de siècle n.e sera par exemple pas ici évoqué, car nous avons souhaité régler la focale sur son œuvre de pionnier en matière de littératures populaires et de culture médiatique.

Charles a en effet été l'un des premiers, dès son mythique Production de l'intérêt romanesque (1973) - dont l'ombre tutélaire de « dieu caché » théorique est évoquée non sans humour ci-après par Marie-Eve Thérenty dans sa présentation de l'article « De la couverture illustrée du roman populaire» - à s'aventurer, en découvreur de corpus occultés et en pisteur des signes, dans les territoires sauvages du « populaire infiniment parlant» (pour reprendre le titre-choc de sa contribution au colloque de Limoges de 1995, reproduite ici même). Dans cet ouvrage fondateur, salué à l'époque pour sa radicalité, Charles s'écartait des lectures transcendantes et des conceptions axiologiques de la littérature, pour embrasser un vaste ensemble de romans publiés sur une période de dix ans (la décennie 1870-1880) et, croisant analyse formelle et histoire de l'idéologie, il montrait que les œuvres manifestaient toujours dans leur structure narrative une dynamique de remise en ordre des forces transgressives du récit, annonçant déjà ce que Marc Angenot décrira quinze ans plus tard comme le discours social, cet ensemble de représentations doxiques, de déroulements de récits attendus et de systèmes axiologiques permettant à une société d'être unie par des valeurs et par 
des topiques communes. Il développera à partir de cet ouvrage germinatif plusieurs de ses concepts essentiels, comme celui des "créances». Mais il en tirera surtout une volonté de s'ouvrir à tous les types de textes, en dehors des hiérarchies instituées, en multipliant les recherches sur des productions obscures, des formes marginales et des domaines jusqu'alors délaissés par les études universitaires, comme la littérature populaire, la photographie, l'illustration ou les productions humoristiques.

Chacun à leur manière, Paul Bleton, Jan Baetens et Marc Lits donnent ainsi un coup de chapeau à l'originalité de la démarche de Charles Grivel, dont un regard rétrospectif distrait pourrait mal mesurer la portée innovante et même iconoclaste en son temps. Depuis une vingtaine d'années, et a fortiori depuis une dizaine d'années, les études sur les formes et les usages des récits multimédiatiques se sont en effet à un tel point multipliées qu'on pourrait en oublier que dans les années 1990 il était rare pour un « littéraire » de déroger à la poétique textuelle pour s'interroger sur l'impact et les enjeux de l'intermédialité sur les pratiques de lecture. Or Charles fit plus que se risquer vers de telles contrées encore peu balisées théoriquement, il en fit l'un de ses territoires de chasse de prédilection, attentif à la matérialité des supports, insistant notamment, par une dilection avouée et assumée pour le visuel, sur le retentissement dans l'imaginaire du lecteur-spectateur de l'image. Certaines de ses hypothèses, formulées sous forme d'aphorismes ou placées au cœur de son ouvrage Fantastiquefiction (1992), mirent aussi judicieusement en lumière la tension fondatrice, matricielle en régime médiatique, entre narration et figuration.

5 Bien sûr Charles fut aussi un pionnier des recherches en littérature populaire et cultures médiatiques, non seulement via ses travaux, mais aussi par son activités dans les réseaux de chercheurs qui se mettront en place au cours des années 1980. Il a été parmi les premiers chercheurs à s'investir au sein de l'Association des Amis du roman populaire, qu'il présidera quelques années; il a participé au lancement de la revue Tapis franc, spécialisée dans l'étude des littératures populaires, puis à celui de Belphégor, élargissant le propos aux cultures médiatiques. Il a enfin contribué à la fondation du réseau des chercheurs en Littératures populaires et cultures médiatiques, devenu l'actuelle association LPCM. Au sein de ces différents ensembles, il a joué un rôle intellectuel majeur, avec son goût de la polémique, du paradoxe, et d'une réflexion se voulant toujours hétérodoxe. Plus généralement, sa pensée se caractérisait par son refus des partitions canoniques et des exclusions, des dichotomies piégeantes fussent-elles pensées comme des progrès vers une neutralisation axiologique dans l'étude rigoureuse de corpus naguère stigmatisés -, qu'il rejetait comme autant de mutilations à la complexité du plaisir nourri des mots. «Je suis, ainsi, populairement fébrile. " déclarait-il, toujours dans " Populaire, infiniment parlant ». Et d'enchaîner : "Je constate un mouvement pendulaire. Il n'y a pas, d'un côté, le littéraire, et, de l'autre quelque chose qui ne le serait pas, le para - ou le péri - de ce qui serait inversement le haut, un impropre combiné à ce qui serait son mieux - et son gage mais seulement deux états de jouissance unique, tour à tour acquiesçante et refoulée. » Il aurait pu dire avec Jérôme Bourdon qu'il est salubre intellectuellement, sous peine d'hypocrisie ou de schizophrénie d' " accepter qu'en matière de culture, nous sommes tous peuple, tous populace, ou, pour employer un mot qu'il faudrait, avec Paolo Virno (2002), restaurer : multitude $»{ }^{1}$

6 Que cette jouissance procurée par le «populaire [...], matière mère » le soit par le ressassement et la stéréotypie, qu'elle fasse son miel de la sérialité, incubateur des jeux 
auctoriaux et lectoraux, Grivel l'a affirmé très tôt («La répétition est belle. Je ne demande que ça: que ça continue et recommence. [...] Un désir de pérennité trouve enfin à se satisfaire, un désir de redondance et de rengaine »), mais sans assimiler comme l'avait fait Umberto Eco dans ses articles précurseurs recueillis dans Il superuomo di massa (De Superman au Surhomme) cette jubilation aux «fastes de la paresse infantile $\aleph^{2}$. Alors que les recherches sur les cultures populaires et médiatiques en étaient encore à leurs débuts, et en écho aux travaux contemporains du regretté JeanClaude Vareille, lui-même grand pionnier trop tôt disparu en 1994, Charles Grivel sut affirmer également avec force, au rebours de toutes les visions condescendantes portées par les clercs universitaires sur les lecteurs forcément aliénés de » mauvais genres » captateurs, que la littérarité du populaire engageait nécessairement un jeu avec le second degré. Écoutons-les : Grivel, en 1995 : « La littérature, la bonne, à tous les étages, comprend dans son principe la distance vis-à-vis de ce qu'elle énonce : elle est ironique et s'emploie à caricaturer aussi les vérités qu'elle applique.»; Vareille, dans son ultime ouvrage, paru l'année de son décès: «la lecture n'est pas croyance ou distanciation. Elle est croyance et distanciation. [...] 'Populaire' est parfaitement compatible avec 'distancié'; peut donc dans le même temps impliquer une esthétique carnavalesque de la dérision et du retrait, et une esthétique expressive de fusionparticipation - ce qui réunit les deux versants canoniques des mentalités populaires $»^{3}$. De tels textes montrent combien des critiques comme Charles Grivel et Jean-Claude Vareille ont contribué, dans le domaine des études littéraires et médiatiques, à ce tournant vers les usages des textes appelé de leurs vœux par des théoriciens comme Richard Hoggart en Grande-Bretagne et Jean-Claude Passeron en France. C'est ce choix qui caractérise par exemple l'approche du fantastique qui fut celle de Charles. A une époque où dominaient les conceptions formalistes du genre, il refusera d'en limiter le périmètre, posant avant tout la question de l'effet produit sur le lecteur ou le spectateur - peur, inquiétude, trouble - et sur son propre sentiment face aux œuvres, ce qui le conduira à prendre en compte une multitude de textes qu'une lecture canonique du genre aurait écartées (Fantastique-fiction).

7 Charles a donc été indubitablement, on l'aura compris, un grand praticien autant qu'un théoricien de la lecture comme jeu du désir et pratique d'une jouissance polymorphe. Dans son texte d'introduction à " Populaire, infiniment parlant ", Lise Dumasy pointe ici avec justesse cette posture fondatrice d'une démarche profondément subjective face aux textes, qui fait pendant au textualisme barthésien, et on ne pourra que l'approuver à la (re)lecture de l'entretien avec Marc Lits publié dans la revue Textyles, ou les deux contributions aux colloques de Limoges de 1998 : «Le passage à l'écran. Littératures des hybrides» ou de Leuven en 2003: "Photocinématographication de l'écrit romanesque». Que cette jouissance grivélienne procède d'une formidable pulsion scopique, soulignée de concert par Paul Bleton et Lise Dumasy, et s'apparente aux délices d'un voyeur, ne fait par ailleurs guère de doute, puisque l'intéressé revendiquait haut et fort cette fascination pour l'image, de sa stase la plus matérielle, celle de l'illustration ou du roman-photo, jusqu'à sa métastase en cosa mentale s'ouvrant sur la scène primitive. Voyez les dernières lignes de son article, au titre benoîtement anodin pour une fois, "De la couverture illustrée du roman populaire». Et que l'image soit pour Grivel l'incubateur textuel et le déclencheur psychique du fantastique est tout aussi manifeste, Marc Lits le rappelle à bon escient dans sa présentation de l'entretien paru dans Textyles. 
8 Mais cet intérêt pour l'image, pour la photographie, le cinéma, pour les petits livres populaires ou leur couverture, manifeste aussi une attention à la matérialité des œuvres et à leur fonctionnement comme dispositifs produisant du sens. Ce n'était pas seulement par bibliomanie que Charles collectionnait ouvrages anciens et curiosités de l'édition, mais aussi par intérêt pour les objets et pour les modes de lecture qu'ils induisaient. Aussi ses interventions tournaient-elles parfois au cabinet de curiosité : descriptions de romans illustrés de photographies posées, ciné-romans, métamorphoses de personnages populaires, formes variées de la «littérature des hybrides» (pour reprendre le sous-titre de l'article présenté par Paul Bleton) devenaient la source d'une promenade spirituelle laissant découvrir, au détour du chemin, de profondes intuitions sur l'expérience des œuvres sous toutes les formes, y compris les plus marginales.

9 Mu par son plaisir, l'explorant dans ses tréfonds, Charles Grivel a par là même déployé une démarche profondément originale, volontiers hétérodoxe sinon hérétique vis-à-vis des courants dominant le champ théorique des études littéraires. Ni structuraliste, ni sociocritique, il prit toujours un malin plaisir à prendre à revers les taxinomies en vogue et les tentatives de totalisation théorique, pensant à partir de la marge, comme le marquent Marie-Eve Thérenty ou Denis Mellier. Ce syndrome de sécession systématique put l'amener parfois à cultiver des positions un brin provocatrices, comme par exemple lors du mémorable débat préparatoire à la rencontre de Limoges du printemps 2003 sur "Culture médiatique et genres", où il affirma tout de go péremptoirement "On écrit hors genres", dans une radicalité généro-sceptique que plusieurs des contributeurs du présent numéro contestèrent frontalement.... Mais l'ami Charles, c'était l'une de ses marques de fabrique, n'aimait rien tant que de faire claquer une formule, de ferrailler pour « remettre l'évidence en chantier» - pour emprunter en le détournant un vers d'Aragon -, fût-ce au prix d'une dénégation acrobatique...

Charles Grivel, par delà sa posture - au sens de Jérôme Meizoz - facétieuse de savant fou, collectionneur invétéré et amateur de cabinets de curiosités littéraires, fut aussi, sous ses dehors et en son for intérieur d'original, un immense érudit (Jan Baetens le signale ici sans détours dans sa présentation de « Photocinématographication de l'écrit romanesque »), adossant ses fulgurances à une connaissance experte des corpus qu'il scrutait et mettait en coupe réglée. Vittorio Frigerio, dans son commentaire de l'article "Alexandre Dumas. Le parler noir », souligne ainsi que "la démonstration se fait en deux temps, et [que] sa construction renverse adroitement les schémas traditionnels. Longue et agréable promenade dans le jardin des impressions d'abord. [...] Discours tout en dérivations, en méandres, en détours, au milieu duquel les affirmations qui structurent la démonstration entière s'érigent épisodiquement, piquets qui marquent le chemin, ou alors, si on veut, éclats inopinés de trompette. Et ensuite, massivement, systématiquement, l'avalanche des preuves, la lecture indiscutable, logique et exemplaire des œuvres.» Grivel ne s'est pas toujours astreint à fournir ainsi explicitement ses preuves, mais on ne doutait guère qu'il les gardait dans sa manche, en bon joueur, savourant ses effets possibles, en virtuose de la parodie au point qu'Alain Vaillant propose ici même de lire l'article métadiscursif « Le retournement parodique des discours à leurres constants » comme un autoportrait...

11 Parmi les miroirs d'encre qu'il fréquentait, Grivel a eu plusieurs amours : le récit imagé, le fantastique (Denis Mellier souligne dans sa contribution à quel point Charles Grivel permit avec quelques autres ténors de repenser le genre), le naturalisme saisi par la 
décadence... mais aussi (surtout?) Alexandre Dumas, "l'homme 100 têtes" pour reprendre le titre génial de son ouvrage de 2008. Ici même de fervents dumasiens, comme Vittorio Frigerio dans sa présentation du «Parler noir », ou comme Sarah Mombert, rappellent combien Charles Grivel aima Dumas, le pénétra, s'en pénétra, jusqu'à peut-être l'inventer. Ici encore, la piste de la négritude et de la stigmate de l'origine (celle du sang-mêlé), tout à la fois comme trait explicatif de l'œuvre de Dumas et de sa posture dans le champ littéraire et artistique tient de l'intuition géniale que Charles Grivel aura décliné dans plusieurs articles avant d'en faire son livre ultime. Selon lui, la mise en scène d'un moi omniprésent et le goût pour les personnages démesurés est une manière de conjurer la place impossible, dans les Lettres, pour celui qui reste un nègre; quant à sa boulimie de création, jusqu'à la désinvolture, elle correspondrait à une quête sans fin parce qu'insatiable pour se faire un nom - en un sens, se blanchir. Pour contrer la neutralisation de ce trait dans la critique, Charles Grivel doit traquer les signes, épousant dans son écriture cette logique d'exploration en cercles concentriques, dans une écriture de la séduction autant que de la conviction ${ }^{5}$.

Et de fait, on ne saurait évoquer Charles Grivel, sa vie, son œuvre... même de manière cavalière et par saillies, comme il aimait à procéder lui-même, sans reconnaître en lui le styliste, habité du démon de l'écriture, en un troublant cousinage avec l'auteur de $\mathrm{S} / \mathrm{Z}$ ou de Fragments du discours amoureux. Grivel, homme d'oralité spectaculaire, lorsqu'il passait à l'écran de l'écriture, s'avérait en effet un artiste de la parole. Jan Baetens identifie par exemple au cœur de son style singulier son penchant pour les procédés intriqués de la synonymie et de l'amplification, et repère sa dilection pour la métaphore comme clef argumentative. L'écriture de Grivel, friand de néologismes, d'inventions lexicales et de maximes inédites de son cru, frappe indéniablement par son goût de la formule armée jusqu'à l'aphorisme, de la pointe, en un mot par sa préciosité, aussi stimulante que provocante, jusqu'à verser parfois dans l'abscons comme le marque Lise Dumasy en toute amitié.

13 Car fondamentalement, un texte de Grivel, «toujours trop elliptique, trop dense, trop erratique - à tous égards, très éloigné des normes actuelles du discours universitaire » pour reprendre les mots d'Alain Vaillant, prétend nous donner à penser, comme le rappelle Lise Dumasy, fût ce au prix d'une certaine opacité oraculaire, d'un refus délibéré de l'ordonnancement logique habituel du raisonnement hypothético-déductif, d'un torpillage par un verbe inventif de la linéarité démonstrative, d'un refus délibéré d'étayer son propos par des références à d'autres travaux de la communauté des clercs ès littérature. Gonflée de la subjectivité d'un lecteur-voyeur, l'écriture de Grivel est donc d'abord et avant tout une écriture du « je », qui se met en scène discursivement, qui ente ses propositions et positions théoriques sur une universalisation sans complexes de son point de vue. Lire Grivel, c'est se frotter à une pensée foncièrement essayistique, qui balaie l'imitation des protocoles scientifiques, et nous propose de petits traités fragmentaires et fragmentistes, avec la force d'une rhapsodie. Nous espérons que ce numéro de Belphégor démontrera que cette parole vive reste d'actualité. 


\section{NOTES}

1. Jérôme Bourdon, , «La télévision et le peuple, ou le retour d'une énigme », in Hermès 42, «Peuple, populaire, populisme », CNRS Éditions, 2005,p 117.

2. Umberto Eco, dans son article "Le mythe de Superman" datant de 1962, écrit par exemple: « N'est-il pas naturel que le jouisseur cultivé - qui, dans ses moments de tension intellectuelle, attend du tableau informel ou du texte sériel qu'il stimule son intelligence et son imagination aspire lui aussi, dans ses heures de détente et d'évasion (salutaires et indispensables ), aux fastes de la paresse infantile, et demande au produit de consommation courante de l'apaiser par une orgie de redondance? " Umberto Eco, "Le mythe de Superman », De Superman au Surhomme, traduction française par Myriam Bouzaher, Paris, Grasset, 1993, p. 161.

3. Jean-Claude VAREILLE, Le Roman populaire français (1789-1914) - Idéologies et pratiques, Limoges / Québec : PULIM / Nuit Blanche Éditeur, Littératures en marge, 1994, p 197.

4. Voir dans le numéro 3, volume 1, décembre 2003, du premier Belphégor, les articles rendant compte de l'atelier de réflexion tenu à Limoges au printemps 2003 dans le cadre de la Coordination internationale des chercheurs en Littératures populaires et culture médiatique, " ancêtre » de l'actuelle association LPCM. http://dalspace.library.dal.ca/bitstream/handle/ 10222/53209/03_01_LPCMLI_debatp_en.pdf?sequence=1\&isAllowed=y ou encore http:// dalspace.library.dal.ca/bitstream/handle/10222/50600/03_01_Migozz_rappor_fr_cont.pdf? sequence $=1 \&$ is Allowed $=\mathrm{y}$

5. On se reportera au compte-rendu qu'avait publié Vittorio Frigerio dans Belphégor, 2008, 1 http://dalspace.library.dal.ca/bitstream/handle/10222/31464/

frigerio_v_belphegor_2008l_fr.pdf?sequence=1 